\title{
Oil Recovery in Heterogeneous Terrigenous Reservoirs
}

\author{
Bakraev M.M. \\ The department of DDEOGF \\ Grozny State Technical Oil University \\ Grozny city, Russia Federation \\ mubarik@yandex.ru \\ Gazabiyeva Z.Kh. \\ The department of DDEOGF \\ Grozny State Technical Oil University \\ Grozny city, Russia Federation \\ anak-777@mail.ru
}

\author{
Mollaev R.Kh. \\ The department of DDEOGF \\ Grozny State Technical Oil University \\ Grozny city, Russia Federation \\ rualanbek47@yandex.ru \\ Umaev A.A. \\ The department of DDEOGF \\ Grozny State Technical Oil University \\ Grozny city, Russia Federation \\ arbium@rambler.ru
}

\begin{abstract}
This article deals with the issues of enhanced oil recovery in terrigenous reservoirs characterized by structural heterogeneity in both the section and along the strike. As a working oil displacing agent, high-pressure gas is used, which is more effective than water-based solutions. The mechanism of the formation of the transition zone in the area of gas mixing with oil is shown and the empirical dependence of the sizes of the transition zone on the length of the linear displacement section and the viscosity ratio of oil and gas in reservoir conditions is established. Information is given on testing a high-pressure gas injection method in the conditions of the Goit-Kort field (CR). It is shown that the introduction of the method allowed to increase the current oil production and oil recovery coefficient in the field.
\end{abstract}

Keywords - reservoir, heterogeneity, phase transition, high pressure gas, oil recovery factor, reservoir, transitional zone, porous medium

\section{INTRODUCTION}

Oil recovery from reservoirs of most fields rarely reaches $50 \%$ of the initial hydrocarbon reserves and the growth of production in our country and abroad leads inevitably to an increase in residual oil reserves.

In deeply located low-permeable and lithologically heterogeneous formations, where the efficiency of waterflooding is often insufficient, special research and development of new methods for enhanced oil recovery using agents capable of more efficiently displacing oil from voids, penetrating into dense rocks and not interacting with them is required.

The most famous method in the world and domestic practice of oil production at present is the process of displacing oil from reservoirs by hydrocarbon gases under high pressure.

The increasing role of gas in enhancing oil recovery determines the need to deepen research into the mechanisms of formation of the transitional zone and the process of mixing oil under high pressure.

\section{ANALYSIS OF THE RESEARCH}

The study of the heterogeneity of reservoir properties of productive reservoirs is of great importance both in the design of the development of oil deposits and in the process of their exploitation.

At the present stage of development of the oil industry, along with the involvement in the operation of new fields, the methods of increasing the degree of oil recovery in the longterm developed deposits, including by pumping hydrocarbon gases under high pressure, are becoming increasingly important.

This issue is of particular importance for areas in which oil deposits are associated with terrigenous deposits, productive reservoirs of which are characterized by significant lithologicphysical variability.

It is obvious that the problem of heterogeneity of productive reservoirs should be solved in a complex with the involvement of data on geology, physics of the formation and hydrodynamics. Basic information about reservoir heterogeneity can only be obtained by geological studies and, first of all, by studying the conditions of sediment formation and the subsequent impact of diagenetic processes on them [1, 2].

Heterogeneity of oil-bearing reservoirs by permeability has a great influence on the course of development processes of deposits and their results. By the nature of its manifestation, three main types of heterogeneity of reservoirs can be distinguished.

1. Heterogeneity associated with the stratification of a single horizon on a number of reservoirs or interlayers, widely developed in area. The study of 
such heterogeneity can be carried out by zonal correlation of layers and drawing up of zonal maps.

2. Heterogeneity due to partial replacement of porous rocks with clays, dense siltstones, argillites, etc., and development, thus, within the horizon of wedged porous interlayers. In this case, to study the nature of reservoir heterogeneity, special attention should be paid to the construction of isopach maps.

3. Heterogeneity associated with a sharp change in the reservoir properties of the horizon across its entire thickness (or in part of this capacity) due to facies variability.

In his position of heterogeneity are divided into regional, central, and areal heterogeneity.

The study of the position of heterogeneity in the productive horizon is important when choosing a development system and measures for the impact on the reservoir [1]. In the development of deposits by injecting gas under high pressure, it is necessary to take into account the heterogeneity of the reservoir for a more accurate assessment of the process efficiency.

The degree and nature of the influence of reservoir heterogeneity on the efficiency of oil displacement of gas under high pressure is estimated according to experimental and field research. Consider the above, for example, the development of the Goit-Kort field, the productive deposits of which are associated with the XXIII stratum of karaganchokrakskij age.

At present, in the section of the XXIII stratum, the GoitKort field of the Chechen Republic, VI packs of sandy interlayers have been identified [3]. According to the analyzes of cores and field geophysical studies of wells I, II and IV, the interlayers have better reservoir properties than III, V and VI interlayers, and their greater distribution within the deposit. In separate wells I and II, the interlayers are additionally divided into 2 layers. Almost everywhere in the areas III and IV, the interlayers merge into one interlayer with the exception of individual wells, in which a clayey section is noted between them. In most cases, layers V and VI are considered together. Within most of the area they are replaced by clay rocks.

Based on the processing data of the complex geophysical materials, the effective oil-saturated thicknesses of individual sandy layers of the XXIII reservoir were determined by wells. The area-weighted average effective oil-saturated thickness for individual sandy layers is $0-19 \mathrm{~m}$ (on average, about $5 \mathrm{~m}$ ).

The effective total oil-saturated sediment thickness of the XXIII reservoir varies from 0 to $46.5 \mathrm{~m}$, averaging $15.43 \mathrm{~m}$. The total thickness of the reservoir decreases in the western and south-western directions. The permeable sandy layers of the XXIII reservoir in the western direction are completely replaced by clays, forming a lithological boundary.

The heterogeneity of the XXIII reservoir deposits was estimated by the area of the section based on data from the processing of geological field materials: electric logging diagrams, cores, thickness of sandy layers in wells. Were also used the values of effective thickness in the wells, obtained according to the processing of the full complex of geophysical materials for 44 wells of the main (western) section of the reservoir. Interlayers with a porosity of more than $10 \%$ and oil saturation of more than $50 \%$ were distinguished as effective. To study the heterogeneity of sediments of the XXIII reservoir within the area, maps of reservoir distribution along the I, II, III + IV and V + VI sandy interlayers were constructed.

The reservoir rocks I and II layers are common in most of the area. The zone of absence of a collector is marked in the western part of the area. Small areas of lack of reservoirs are noted in areas of individual wells. Practically within the entire area, with the exception of the area of wells No. 73, 116, 117, reservoir rocks of III + IV sand layers are common.

The reservoir rocks of the $\mathrm{V}+\mathrm{VI}$ sand interlayers are distributed within the central part of the area. In the western and eastern directions, sand interlayers are replaced by clayey siltstones and clays.

The pinching out of individual sandy layers of the XXIII reservoir and an increase in the clay content of the sediments is also observed in the direction of the arch part of the structure. To assess the heterogeneity of the filtration properties of sandy layers of the XXIII reservoir, data on determining the permeability values of the cores were analyzed. Small values of permeability of sandy layers are noted in the central part of the area. In the western direction, the permeability of sediments decreases [4].

In order to quantify the heterogeneity of sediments of the XXIII reservoir, such heterogeneity indicators were calculated, such as the coefficient of dissection and the coefficient of sandiness.

The ratio of sandiness, showing the ratio of the effective thickness to the total thickness, was determined by 44 wells and averaged 0.226 . The coefficient of dissection, showing the ratio of the number of effective interlayers of sandstone, summarized over all wells, to the number of wells was 6.4. The obtained value of the coefficient of dissection shows that the XXIII reservoir is largely heterogeneous.

The saturation porosity of sediments of the XXIII reservoir, determined by core samples, ranges from 2 to 17.4 , averaging 9.4\%. According to 227 core definitions, the average permeability is $43 \cdot 10-15 \mathrm{M}^{2}$, varying from 0 to 144 . $10^{-15} \mathrm{M}^{2}$. According to the data of field geophysical studies of 55 wells, the saturation porosity varies from 12.1 to $22.3 \%$. On average, the deposit is $17.5 \%$.

According to laboratory data from core studies, oil saturation varies from 23 to $82.6 \%$, averaging $58.6 \%$ of the deposit. The value of oil saturation, determined by field geophysical studies, is $64.7 \%$. To evaluate the permeability parameter, hydrodynamic well studies were also used. A total of 16 wells were investigated. Permeability according to these studies varies from 4 to $64 \cdot 10^{-15} \mathrm{M}^{2}$, averaging $23,7 \cdot 10^{-15} \mathrm{M}^{2}$.

As can be seen from the above, XXIII reservoir is characterized by low permeability, substantial heterogeneity and shale. The process of oil displacement by water in such conditions is very difficult, and therefore attempts to master the injection of water into the XXIII reservoir were 
unsuccessful. In accordance with the criteria of applicability and taking into account the results of laboratory studies, injection of high-pressure hydrocarbon gas into the reservoir of the hydrocarbon gas was proposed to enhance the oil recovery of reservoir XXIII of the Goit-Kort field.

Experimental studies [4, 5] of the displacement of paraffinic, naphthenic and aromatic hydrocarbons with highpressure gas show that the process efficiency under comparable conditions depends on the nature of the displaced agent and the degree of its miscibility with the displacing agent.

The use of well-known modeling methods, including the construction of triangular diagrams for describing physical phenomena that occur in a porous medium during the formation of a transitional displacement zone, makes it possible to trace to a certain extent qualitatively the process [5, $6]$.

To create computational methods for evaluating the behavior of hydrocarbon systems at high pressures, the use of these models did not bring any significant results and still the main way to study the physicochemical phenomena occurring in the hydrocarbon system at high pressures is, especially in engineering practice, experimental studies .

Experiments on linear reservoir models with specific fluids determine the pressure, the excess of which does not give a significant increase in displacement efficiency. This pressure at a high absolute level of displacement efficiency is, under these conditions, sufficient for the formation of a transition zone mixed with oil and gas. In the same way, the minimum required content of intermediate components in the injected gas is determined at the established pressure and reservoir temperature.

According to modern concepts, in the formation of a transitional displacement zone between oil and compressed gas, there is no area in the reservoir where "preparatory" processes of component exchange between the displaced and injected agents would occur.

The formation of the transition zone begins in the cross section of the contact of oil and gas and is carried out continuously in the future with linear movement of fluids in the reservoir.

For a theoretical description of changes in the size of the transition zone, some authors V.N. Nikolaevsky, R.A. Bagov, N.L. Rakovsky, A.Yu. Namiot [7, 8] use the laws of diffusion intermixing of mixing agents.

Close to engineering practice and in terms consistent with our studies of the displacement of oil by compressed gases, the question of mixing of mutually soluble liquids with different viscosity was solved by Zheltov Ju.P, which took as the main hypothesis the dependence of the mixing coefficient on the viscosity gradient in the zone of the solution.

BIn related areas of industrial technology [9], special cases of solving the diffusion equation using modern techniques sufficiently describe the process of impurity scattering, without taking into account its viscosity characteristics.
Experimental studies to a certain extent reflect the real conditions of mixing inter-soluble fluids as they move in a porous medium. Laboratory experiments using linear reservoir models of various lengths allowed us to obtain an empirical relationship to determine the size of the transition zone when oil is displaced by high-pressure gas

$$
\imath=2 \cdot \sqrt[3]{A \cdot L^{2}} \cdot\left(0,1+0,23 \sqrt{\mu_{0}}\right)
$$

где $\ell$ - transition zone length, м;

$A$ - linear scale correlation coefficient, ;

$L$ - the length of the linear section at which the displacement occurs, $\mathrm{M}$;

$\mu_{o}$ - oil viscosity to gas viscosity ratio at reservoir conditions.

This formula characterizes the change in the transition zone not only along the length of the displacement section, but also depending on the viscosity properties of the filtering agents.

The obtained dependence can also characterize the mechanism of the process in a radial flow, i.e. mixing of fluids, as they move along the linear coordinates of the axisymmetric direction.

The maximum coincidence of the results of theoretical studies and experiments by agents intermingling with it, if the velocity of the linear motion of fluids is commensurate with the speed of diffusion penetration of the displacing agent can be expected in the study and implementation of the process of oil displacement [10].

This conclusion makes it possible to single out a promising direction in the development of methods for describing the mechanism of mixing inter-soluble fluids as they move in a homogeneous void medium. Such a direction could be the development of sufficiently general similarity criteria and criterial equations characterizing the process.

Analysis of the theoretical study of the process and experimental studies of oil displacement by hydrocarbon gases on linear models made it possible to evaluate the oil displacement efficiency as applied to specific reservoir conditions of a number of fields (Ozek-Suat, Goit-Cort), to determine the required level of gas injection pressure in real conditions, to describe the oil mixing mechanism and gas in a porous medium at a linear flow.

Over the decade, about 1.5 billion $\mathrm{m}^{3}$ of gas was injected into the reservoir, which made it possible to increase the current reservoir pressure, transfer a number of wells to the fountain method of operation, significantly increase the rate of oil production and oil recovery. During this period, more oil was produced than in the entire previous field operation; a significant amount of gas was saved for future rational use.

Analysis of the current state of development has shown that the extreme heterogeneity of the XXIII reservoir has complicated the implementation of the process, but it has not become a fundamental obstacle to the enhanced oil recovery of complex fields using gas methods. 
Analysis of the enhanced oil recovery process of deeplying low-permeable heterogeneous reservoirs by pumping high-pressure hydrocarbon gases (Goit-Cort, Ozek-Suat) showed that oil production rates achieved during the period of depletion of natural reserves of reservoir energy can be easily exceeded during injection in the reservoir of compressed gas $[11,12]$.

With the introduction of high-pressure gas injection technology of the XXIII reservoir, minor complications were observed associated with gas breakthroughs in individual production wells and an increase in the gas factor. However, these complications were successfully eliminated by selective plugging of highly permeable zones of the reservoir (in particular, sodium silicate solutions), in which, mainly, breakthroughs of free gas occurred.

\section{RESUlTS AND RECOMMENDATIONS}

The theoretical and experimental studies have allowed to obtain the following results:

1. the empirical dependence of the description of the transition zone size for the radial flow in a porous medium is proposed;

2. the dependence of the size of the transition zone on the radius of gas flow in a porous medium with different ratios of the viscosity of the displaced and injected agent is the first approximation of the permanent model of changing the mixing zone in a real reservoir;

3. the introduction of enhanced oil recovery technology by pumping high-pressure gas made it possible to additionally extract a large amount of oil from the XX reservoir of the Goit-Kort field and accumulate the injected gas in the reservoir;

4. the technological scheme of injection of high pressure gas into the reservoir can be recommended for other deposits in similar geological conditions.

\section{CONCLUSION}

Theoretical and experimental studies carried out under heterogeneous, lithologically unsealed reservoirs made it possible to select a working agent for injection into the reservoir to increase oil and oil recovery, substantiate the mixing model of mutually soluble fluids, to obtain an empirical dependence of the size of the transitional displacement zone on the linear flow length and viscosity of the oil and gas in reservoir conditions. Based on the results of the studies performed, the technology of injection of highpressure gas into the terrigenous reservoir of the Goit-Cort field is given. As shown by the results of pilot tests, the method of enhanced oil recovery by pumping high-pressure gas into the reservoir is quite effective and allows improving the technical and economic indicators of reservoir development.

\section{References}

[1] M.A. Zhdanov, F.A. Grishin, E.V. Gordinsky, "Basics of field geology of gas and oil". Moscow: Nedra, 1966, p. 275

[2] A.Sh. Khaladov, A.A. Umaev, V.A. Muskhanov, I.I. Aliyev, M.M. Dudayev, D.A. Islamov, "Study of the oil recovery status of the Mesozoic deposits of the Chechen Republic field and measures for the development of oil production", Proceedings of the Grozny State Oil Technical University, no. 8, Grozny, 2008, pp. 7-10.

[3] A.Sh. Khaladov, Research, development and application of methods to intensify production from the Mesozoic deposits of the Chechen Republic. UFA: BSU, 2009, p. 208.

[4] L.N. Chizhov, V.K. Artukhovich, N.M. Degtyarev, "Assessing the effect of reservoir heterogeneity on the efficiency of oil displacement by highpressure gas. Issues of technology, drilling technology, well testing and development of oil fields", Proceedings of SevKavNIPIneft, no. 26. Grozny, 1977, pp. 69-75.

[5] N.M. Degtyarev, "On the state and composition of the transition zone when oil is displaced by high-pressure gas", Proceedings of Grozny, no. 10. Moscow, 1961, pp. 140-145.

[6] E.R. Hirazov, K.Sh. Yamaletdinova, S.S. Gotz, R.M. Khakimov, V.V. Salyakhov, "Investigation of the diffusion of the components of the liquid phase into the gas and back with the mixing of oil displacement", Problems of collection preparation and transport of oil and oil products, no. 8. Ufa, 2008, pp. 18-25.

[7] R.U. Blackwell, U.R. Rayne, W.M. Terry, "Factore influencing the efficience of miscible displacement", J. Petr. Techn., vol. 1. pp. 1-8, 1959.

[8] A.Yu. Namiot, Phase equal balance in oil production. Moscow: Nedra, 1976, p. 183

[9] V.N. Nikolaevski, Je.A. Bondarev, M.I. Mirkin, Movement of hydrocarbon mixtures in porous operating environment. Moscow: Nedra, 1968, p.192

[10] M.M. Bakrayev, "Physico-chemical methods of monitoring the process of oil displacement by compressed gas", Proceedings of the Grozny State Oil Technical University, no. 4, Grozny, 2004, pp. 158-60.

[11] B.G. Pokusaev, D.A. Kazenin, S.P. Karlov, Yu.N. Skochilova, "Areas of influence of the impurity source in filtration flows", Ecology and Industry of Russia, no. 4, Moscow, 2008, pp.41-43.

[12] V.K. Artukhovich, N.M. Degtyarev, L.A. Bondarenko, "Results of the study of gas injection into the XXIII stratum of the Goit-Kort field. Improving the efficiency of oil production", Proceeding of SevKavNIPIneft, no. 40, Grozny, 1984, pp. 37-44. 